\title{
Dynamic Clamp: Alteration of Response Properties and Creation of Virtual Realities in Neurophysiology
}

\author{
Michael N. Economo, ${ }^{1}$ Fernando R. Fernandez, ${ }^{2}$ and John A. White ${ }^{2}$ \\ ${ }^{1}$ Department of Biomedical Engineering, Boston University, Boston, Massachusetts 02215, and ${ }^{2}$ Department of Bioengineering, Brain Institute, University \\ of Utah, Salt Lake City, Utah 84112
}

\begin{abstract}
Introduction
The vast repertoire of electrical activity displayed by neurons, cardiac myocytes, and various endocrine and sensory cells is the result of membrane-bound ion channels each producing a distinct conductance that facilitates current flux through the membrane. These conductances may be static, or their magnitudes may be voltage or ligand dependent. Dynamic clamp, a term for the various combinations of software and hardware that simulate these conductances, has proven to be a valuable tool for electrophysiologists studying excitable cells. One can think of dynamic clamp as a tool for immersing neurons in virtual reality-inspired experiments. Virtual synapses may be simulated, allowing a biological neuron to communicate with or receive inputs from real and simulated cells. Dynamic clamp can also be used to introduce virtual voltage-gated channels into biological neurons, thus altering the neurons integrative properties. This Toolbox article includes a brief description of how conductances are simulated as well as a discussion of the neurophysiological applications of dynamic clamp and its potential pitfalls. The origins of dynamic clamp, its implementation, and its appli-
\end{abstract}

Received Dec. 1, 2009; revised Jan. 11, 2010; accepted Jan. 13, 2010.

This work was supported by National Institutes of Health Grants R01 RR020115, R01 NS034425, and RC1 NS069033. We thank Dr. Tilman P. Broicher, Dr. Roy M. Smeal, and Dr. Alan D. Dorval Jr. for useful discussions and comments on this manuscript.

Correspondence should be addressed to John A. White, Department of Bioengineering, University of Utah, 20 South 2030 East, BPRB 108, Salt Lake City, UT 84112. E-mail: john.white@utah.edu.

DOI:10.1523/JNEUROSCI.5954-09.2010

Copyright $\odot 2010$ the authors $\quad 0270-6474 / 10 / 302407-07 \$ 15.00 / 0$ cations to cardiac and endocrine electrophysiology have been reviewed elsewhere (Sharp et al., 1993; Prinz et al., 2003; Goaillard and Marder, 2006; Wilders, 2006; Destexhe and Bal, 2009).

\section{Conductance injection}

The current $I$ that flows through a population of channels is governed by the equation $I=G_{\max } a\left(V_{\mathrm{m}}-E_{\mathrm{ion}}\right)$. In general, $G_{\max }$, the maximal conductance, is a constant that depends on the density of channels in the membrane and the speed with which ions can pass through the channel. The reversal potential, $E_{\mathrm{ion}}$, is determined by the concentration of ions inside the cell and in the extracellular space. Therefore, to simulate the current introduced by a membrane conductance, one must be able to continuously monitor the membrane voltage, $V_{\mathrm{m}}$, and determine the fraction of channels that are open, $a$, at each point in time. $V_{\mathrm{m}}$ can be measured with sufficient accuracy by a patch pipette in the whole-cell configuration. For voltage-gated channels, the time evolution of $a$ is typically modeled by a differential equation that describes the kinetics of channel opening and closing in response to changing voltage. For a constant conductance, such as the leak conductance, $a$ is constant and equal to 1 . For calcium-dependent $\mathrm{K}^{+}$and similar channels, the model must account for the molecular species to which the channels are sensitive. Similarly, to simulate channels that depend on binding of a neurotransmitter, one must additionally account for changing ligand concentration to calcu- late $a$. Models of postsynaptic conductances gated by neurotransmitters are typically phenomenological, based on evoked or spontaneous responses measured under voltage or current clamp.

The current produced by this simulated population of channels is subsequently injected into the cell either using a second patch pipette or, if the amount of current to be injected is sufficiently small, with the same pipette used to monitor voltage (Fig. 1). As a result, the magnitude of current flowing into the cell is the same as if a channel with the modeled properties was embedded into the cell membrane. For this reason, simulating channels via dynamic clamp is often referred to as "creating artificial conductances” (Sharp et al., 1993) or a "virtual knockin" (Dorval and White, 2005).

\section{Virtual pharmacology}

Careful voltage clamp studies have been undertaken to quantify the maximal conductance, reversal potential, and dynamics governing the gating of many of the channels typically present in neuronal cell membranes. Although virtual knockins via dynamic clamp using these parameters can be powerful for studying the impact of various channels on the input-output properties of neurons, more sophisticated manipulations can be undertaken as well in which properties of a model channel may be altered to determine which features of the channel impact membrane dynamics.

Dorval and White (2005) provide a good example of such a manipulation. In 


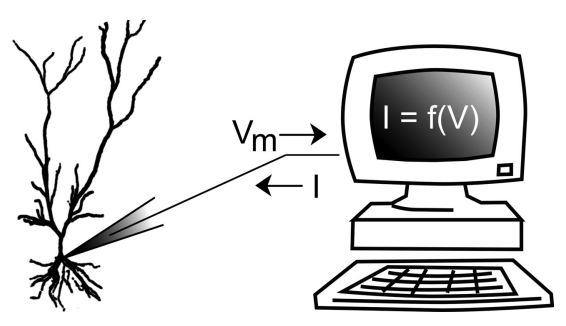

Figure 1. Schematic of the dynamic clamp experimental setup. Voltage of a cell is monitored, typically with a patch pipette, and a feedback current is calculated in real time with a computer or analog circuitry according to a predetermined model. This model may describe a voltage- or ligand-gated ion channel, or some other process. The feedback current is then applied to the cell with the same or a second patch pipette (see Technical challenges).

this study, dynamic clamp was used to show that the stochasticity of the persistent sodium current, $I_{\mathrm{NaP}}$, is essential to its role in the generation of subthreshold membrane potential oscillations and input selectivity in stellate cells of the medial entorhinal cortex (Fig. 2). Although all channels open and close probabilistically, stochastic flicker of $I_{\mathrm{NaP}}$ channels has been shown to be the major source of membrane potential noise in these cells in vitro. This is the result of a relatively small number of $I_{\mathrm{NaP}}$ channels in the membrane coupled with their large single-channel conductance. In this example, $I_{\mathrm{NaP}}$ was blocked using the neuroprotective drug riluzole, abolishing subthreshold membrane potential oscillations. To assess the impact that the stochasticity of the channel, in particular, has on membrane dynamics, the $I_{\mathrm{NaP}}$ channel was then knocked in virtually in either a deterministic form or using a model in which the number of open and closed channels was determined probabilistically at each time step. Subthreshold membrane potential oscillations similar in power to the control were restored after addition of the stochastic, but not the deterministic, persistent sodium channel (Fig. $2 B, C$ ).

Similar virtual pharmacological experiments have been performed in a number of studies to elucidate the role of specific channels. In CA1 pyramidal cells, $I_{\mathrm{NaP}}$ reduces gain, regularizes spike trains, and increases response variability (Vervaeke et al., 2006). Idoux et al. (2006) used dynamic clamp to contrast the spatial distribution of $I_{\mathrm{NaP}}$ and its impact on excitability in type B and type D cells in the prepositus hypoglossi nucleus of the guinea pig, while Hughes et al. (1998) demonstrated the vital role of the hyperpolarization-activated cation current, $I_{h}$, on delta frequency bursts in thalamocortical neurons.

A Riluzole \&
Control Riluzole \&
ODE $g_{\text {Nap }}$
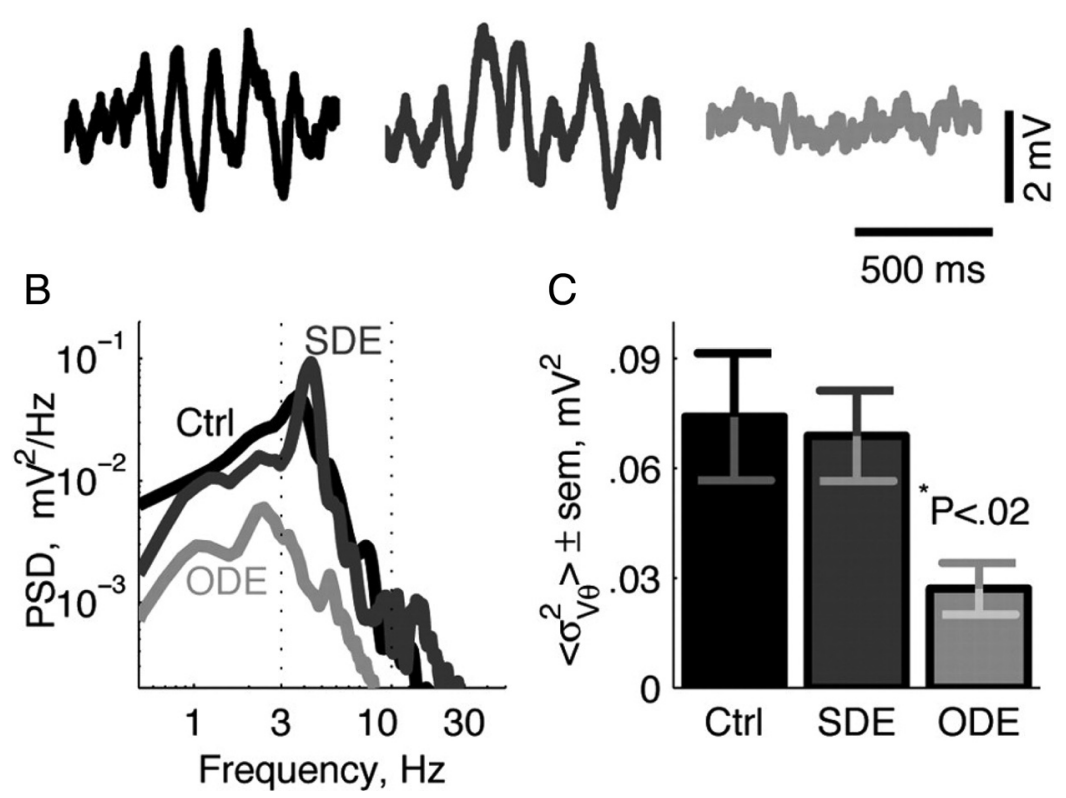

Figure 2. Intrinsic membrane properties associated with theta fluctuations in medial entorhinal cortical stellate cells require channel noise. Reproducing subthreshold theta oscillations in stellate cells in the presence of riluzole $\left(I_{\text {NaP }}\right.$ blocker) requires a noisy representation of $I_{\mathrm{NaP}}$. Raw traces of subthreshold membrane voltage $(\boldsymbol{A})$, power spectral density (PSD) plot $(\boldsymbol{B})$, and power in the theta band $\left(\boldsymbol{C},\left\langle\sigma^{2}{ }_{v \theta}>\right.\right.$, value defined as the integral of the PSD within the theta range-dotted lines) of subthreshold membrane voltage was more closely matched to control when a stochastic differential equation (SDE) was used to implement the $I_{\text {NaP }}$ in the presence of riluzole. Implementation of a deterministic model of $I_{\text {NaP }}$ using a standard ordinary differential equation (ODE) fails to reproduce control levels of power at theta in membrane voltage fluctuations $(\boldsymbol{B}, \boldsymbol{C})$. Asterisk denotes significance using a pairwise $t$ test. Adapted with permission from Dorval and White (2005). Ctrl, Control.

\section{Artificial synaptic input}

Synaptic inputs, represented by stereotyped current waveforms, have been applied to neurons to study their integrative and response properties for many years. However, synaptic inputs introduced in this way do not capture two vital, interrelated aspects of realistic conductancebased inputs. First, conductance inputs interact in a nonlinear manner. For example, the inhibitory action of GABAergic inputs is typically not achieved as a result of large deviations in membrane potential but by "shunting" the current produced by excitatory inputs, thus reducing their impact on the postsynaptic cell. This GABAergic shunting is a direct result of the reversal potential of GABA receptor channels, which is close to resting potential (Hille, 2001). Second, conductancebased synaptic inputs, native or simulated via dynamic clamp, decrease the input resistance of the cell membrane and, consequently, the time constant of the cell, thus reducing the time interval over which inputs are integrated (Paré et al., 1998; Destexhe and Paré, 1999; Destexhe et al., 2003).

In vivo intracellular recordings in awake and anesthetized animals have shown that neurons in the neocortex (Matsumura et al., 1988; Baranyi et al., 1993; Borg-Graham et al., 1998; Paré et al., 1998; Destexhe and Paré, 1999) and other regions of the nervous system (Jörntell and Ekerot, 2006; Berg et al., 2007, 2008) receive a constant barrage of synaptic input. This synaptic activity has been shown to reduce the input resistance and membrane time constant by $50-80 \%$ and to produce a significant depolarization even when the local network is relatively inactive (Destexhe et al., 2003). These findings imply that neurons reside in a qualitatively different state in the intact brain than the relatively quiescent state observed in vitro. Destexhe et al. (2003) have shown that this "high conductance state" can be replicated during in vitro experiments by applying high rates of artificial synaptic inputs to a cell via dynamic clamp (Fig. 3A,B).

Following this approach, it has been shown that the input-output behavior of CA1 pyramidal cells switches from a primarily integrative mode, responding to the average rate of synaptic input under quiescent conditions, to a mode in which they preferentially respond to highly cor- 


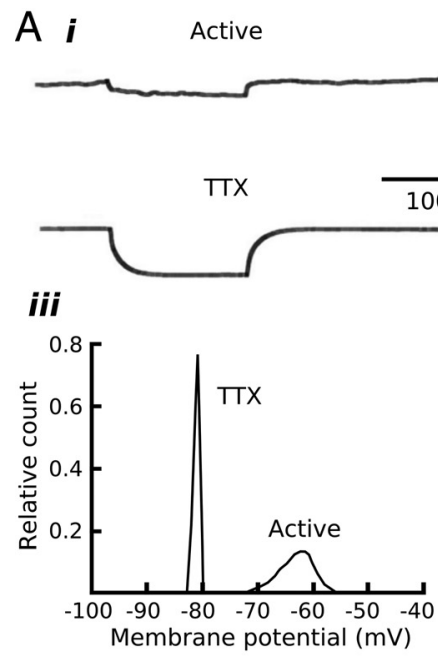

B

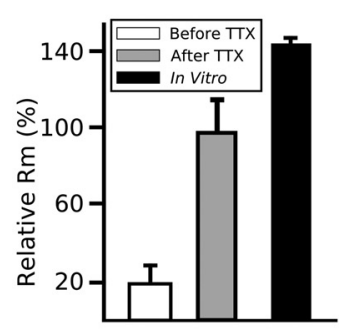

C

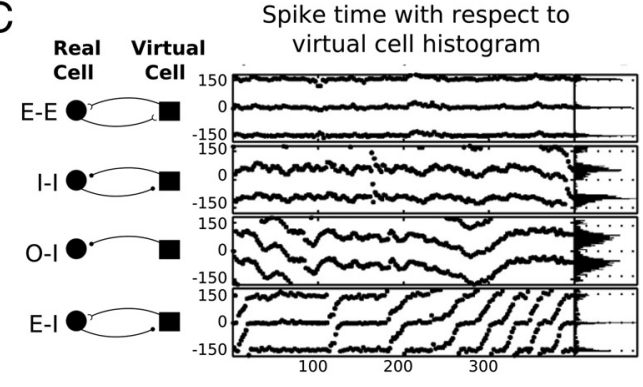

Figure 3. Artificial synaptic conductances introduced via dynamic clamp can be used to simulate in vivo-like conditions. $\boldsymbol{A}$, Examples of raw traces of membrane voltage $(\boldsymbol{i}, \boldsymbol{i i})$ and histograms of membrane voltage distributions (iii, iv) during in vivo activity (i, iii) and in vivo-like conditions reproduced using artificial synaptic activity in vitro (ii, iv). Focal application of TTX near the site of recording in vivo increased the voltage deflection associated with an intracellular current step due to an increase in membrane input resistance caused by the cessation of synaptic activity (iii). Conversely, artificial synaptic activity introduced in vitro can reduce voltage deflections in response to a current step by reducing membrane input resistance (ii). Additionally, membrane voltage distributions are wider under in vivo (active) and in vivo-like conditions due to synaptic activity (iii, iv). $\boldsymbol{B}$, Membrane input resistance is reduced significantly in vivo due to synaptic activity. The bar graphs quantify the relative membrane input resistance $\left(R_{\mathrm{m}}\right)$ under in vivo, in vivo with focal application of TTX, and in vitro conditions. Application of TTX in vivo eliminates synaptic activity and increases $R_{\mathrm{m}}$ to values approximating those measured in vitro. C, Spike time differences between a medial entorhinal cortical stellate cell and an artificial cell simulated using dynamic clamp. Stellate and artificial cells were connected via virtual synapses constructed in dynamic clamp and DC-driving currents were adjusted to generate spike times at 100 ms intervals. The following synaptic connection patterns were used: mutual excitation (E-E); mutual inhibition (I-I); periodic inhibitory drive from the artificial cell with no feedback from stellate cell $(0-1)$; and excitatory drive from the stellate cell with inhibition from the artificial cell $(E-I)$. In the middle section of $\boldsymbol{C}$, the spike time differences are plotted against the spike number. The zero-crossing on the $y$-axis indicates the firing time of the artificial neuron; points represent the relative firing times of the stellate cell to the artificial cell. Spike time values $<0$ ms indicate that the spike in the stellate cell preceded that in the artificial cell. In the right-most section of $\boldsymbol{C}$, histograms of the spike time differences are plotted. $\boldsymbol{A}$ was adapted with permission from Destexhe and Paré (1999) and Destexhe et al. (2001). B was adapted with permission from Destexhe et al. (2003). C was adapted with permission from Netoff et al. (2005).

related inputs in the high-conductance state (Prescott et al., 2006, 2008). A qualitative shift in response properties has also been shown in thalamocortical neurons in the high-conductance state (Wolfart et al., 2005). During dynamic clamp-mediated synaptic barrages, there was no clear delineation between the two states these cells have been shown to occupy in vitro: a quiescent "down state," in which synaptic inputs are reliably relayed, and a burst-firing "up state," in which the cell is relatively insensitive to the spiking of its presynaptic partners. Instead, these cells occupied a range of states intermediate between the up and down states with a commensurate range of input sensitivities.

An advantage to a flexible, real-time, feedback control system like many dynamic clamp implementations is the ability to perform other operations in real time in addition to the simulation of conductances. For instance, the ability to detect action potentials in real time coupled with the capability to simulate synaptic conductance transients allows for the construction of virtually connected networks. In this scheme, two neurons may be recorded simultaneously and an artificial synaptic conductance initiated in one cell whenever a spike occurs in the other. Similarly, one may record from a single neuron and simulate reciprocal connections with one or more in silico neurons whose activity are simulated in real time with the dynamic clamp software, in this way constructing a hybrid network of real and artificial cells. This technique has been applied to study central pattern generators in the lobster stomatogastric ganglia (Harris-Warrick et al., 1995; Sharp et al., 1996; Bartos et al., 1999; Elson et al., 2002; Thirumalai et al., 2006) and to study the manner in which reciprocally connected cells synchronize (Fig. 3C). Hybrid network approaches are also useful for model validation. Replacing a modeled cell in a simulated network with an in vitro recorded cell and observing that the network behavior remains qualitatively similar is an excellent validation that the modeled cell captures the necessary features of the real cell for the study.

\section{Feedback control}

Apart from simulating conductancesboth synaptic and voltage-gated-a flexible dynamic clamp system enables all manner of experiments requiring feedback control. One application that falls into this category is the simulation or stimulation of feedback excitation and inhibition following each action potential in a recorded cell in vitro. In the "reactive clamp" of Fellous and Sejnowski (2003), persistent activity was induced in a spontaneously firing cell when artificial feedback excitatory and inhibitory synaptic conductances were applied to the cell following each spike. More recently, dynamic clamp has been used to control light-activated opsins expressed in fastspiking interneurons (Fig. 4B). When inhibition was stimulated in this way following action potentials in a neocortical pyramidal cell, the pyramidal cell was found to oscillate at gamma frequencies, providing direct evidence that gamma oscillations can be induced by feedback from fast-spiking interneurons, as predicted by theoretical and experimental studies (Traub et al., 1996; Whittington et al., 2000; Atallah and Scanziani, 2009).

Similar to the idea of virtual pharmacology, dynamic clamp has been used to knock in conductances that have no direct biophysical correlate. Fernandez and White (2008) devised an afterhyperpolarization (AHP) reduction conductance as well as a similar AHP enhancement conductance to show that the spike train rhythmicity observed in entorhinal stellate cells can be attributed to the shape of 
A

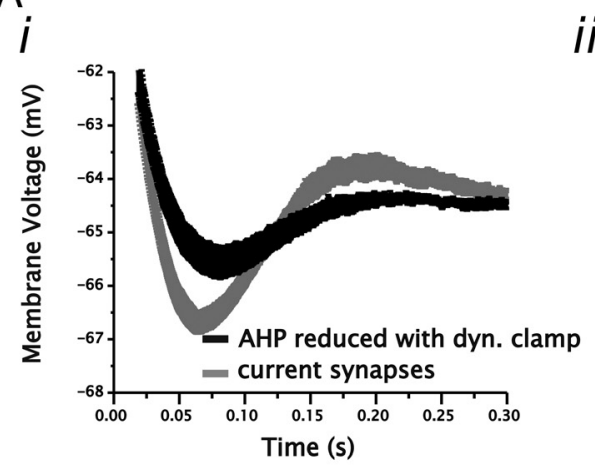

ii

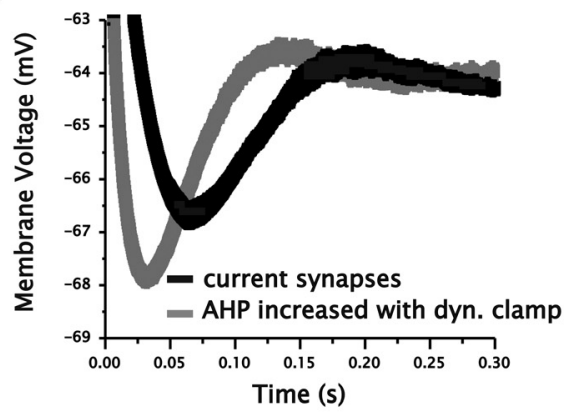

B

i
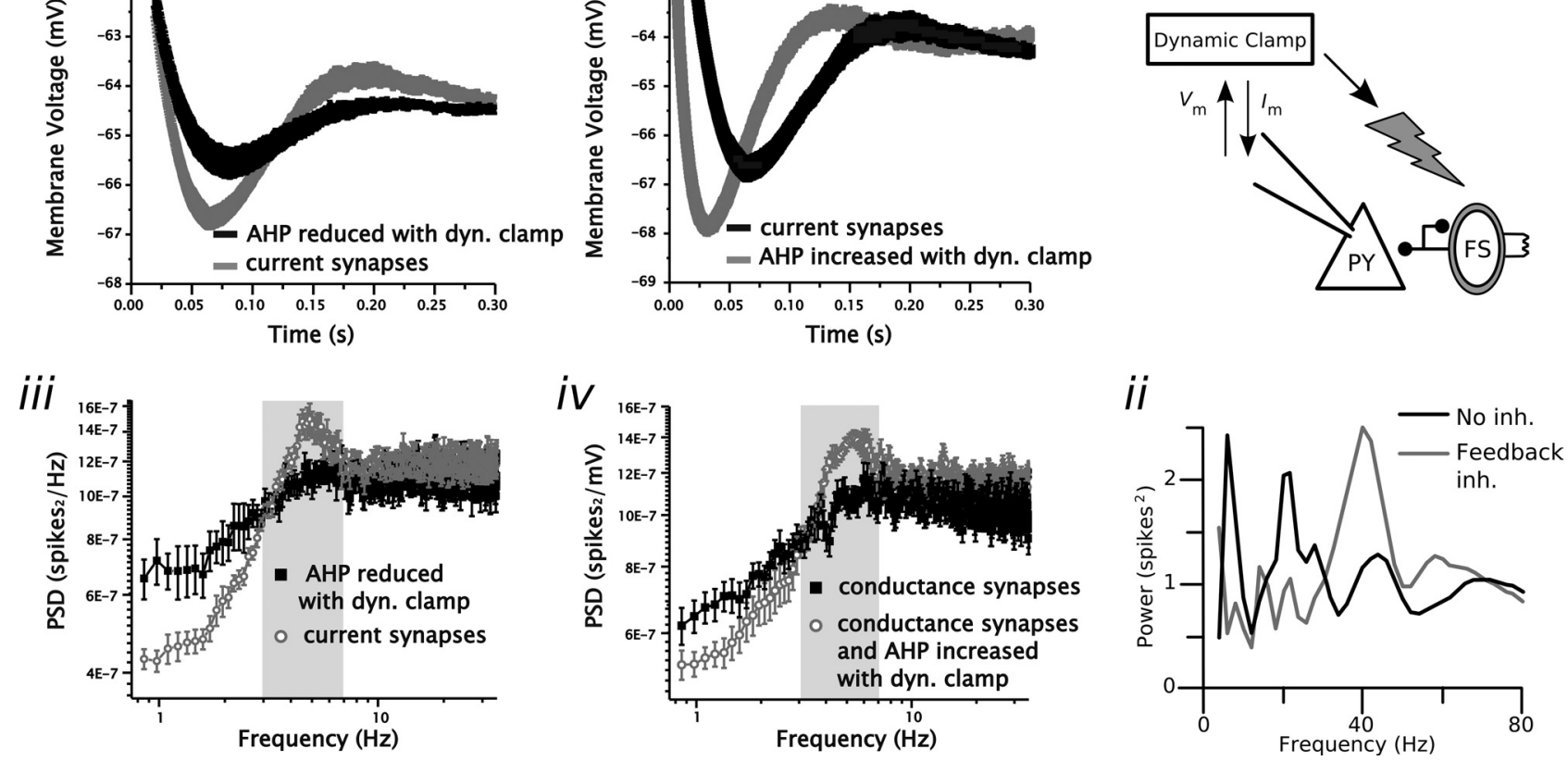

Figure 4. Examples of feedback control implemented with dynamic clamp. A, Manipulation of the spike AHP in medial entorhinal cortical stellate cells using an AHP-reducing (i) or AHPenhancing (ii) conductance introduced via dynamic clamp can be used to regulate the expression of spike train theta oscillations (iii and iv). Under control conditions, the voltage trajectory immediately following a stellate cell spike AHP is associated with a rebound in membrane voltage $(\boldsymbol{i}, i i)$. By changing the amplitude of the AHP using a spike-dependent feedback conductance, the extent of membrane voltage rebound that follows the spike can be manipulated. Reducing the size of the AHP leads to a reduction in the rebound and a reduction in spike train power at theta ( $i$, iii). Conversely, restoring the size of the AHP restores spike train power at theta (ii, iv). B, Feedback inhibition from inhibitory interneurons is necessary for network-mediated gamma ( $30-80 \mathrm{~Hz}$ ) oscillations in neocortical circuits. A photostimulation signal triggered using a dynamic clamp setup monitoring the membrane voltage of a cortical pyramidal cell (PY) can be used to excite a population of interneurons (FS) expressing channelrhodopsin-2. Every time a pyramidal cell elicits a spike a blue light pulse is triggered that leads to excitation of interneurons and subsequent feedback inhibition (inh.) in pyramidal cells. The spike train power spectrum of pyramidal cells shows a peak in the gamma frequency range when the feedback inhibition from inhibitory interneurons is activated using the dynamic clamp-mediated photostimulation (ii). dyn. clamp, Dynamic clamp. $\boldsymbol{A}$ was adapted with permission from Fernandez and White (2008). $\boldsymbol{B}$ was adapted with permission from Sohal et al. (2009).

the AHP (Fig. 4A). These currents are not intended to mimic native membranebound channels but instead simply manipulate the voltage trajectory following an action potential. This manipulation could be implemented just as effectively as an AHP-modifying current, although this would be difficult without a real-time system able to inject current with a very short, consistent delay after an action potential is detected.

\section{Technical challenges}

The electrophysiological prerequisite for implementing dynamic clamp is simple: intracellular voltage must be measured accurately in the presence of applied current. This is not an issue when separate pipettes are used to measure voltage and apply current, but when recording with a single pipette in bridge mode, a certain amount of inaccuracy is inevitable. Although the access resistance-the resistance due to the recording pipette-is typically compensated for with an intracellular amplifier, this compensation is typically only $80-90 \%$ accurate. This im- perfect bridge balance results in a voltage measurement error that is directly proportional to the amount of current flowing through the pipette. This measurement error is typically small for low-resistance patch pipettes passing modest amounts of current. Sharp microelectrodes and highresistance patch pipettes (e.g., those used to record in vivo) introduce considerable inaccuracy, and, although methods exist to minimize this error (Brette et al., 2007, 2008), extra care must be taken to ensure that sufficient accuracy is obtained.

While typically more expensive and less flexible than software-based systems, dynamic clamp systems implemented in hardware are able to update the applied current to a cell nearly instantaneously as voltage fluctuates. Software-based systems require that an analog voltage signal be digitized, some calculation be performed in the computer, and then the correct analog control signal be generated and applied to the neuron. This sequence of events takes a finite amount of time (latency) and is repeated some predetermined number of times per second (up- date rate). The variation in the update period from time step to time step is referred to as "jitter." The effect of update rate, latency, and jitter on dynamic clamp performance and stability has been quantified in detail (Butera and McCarthy, 2004; Raikov et al., 2004; Bettencourt et al., 2008; Preyer and Butera, 2009). In general, the update rate should be sufficient to capture the dynamics of the conductance being modeled as well as the time course of voltage fluctuations. An update rate of $10-20 \mathrm{kHz}$ is typically sufficient for this purpose and is possible on nearly every dynamic clamp system. Simulating a conductance with very fast kinetics, such as the transient sodium channel responsible for the upstroke of action potentials, requires a much higher update rate (Bettencourt et al., 2008). Latency and jitter should be minimized to the extent possible. Conversion between analog and digital signals constitutes another source of error, although this inaccuracy becomes relevant only when dealing with small signals. In practice, dynamic clamp systems have been implemented in a 
number of environments. Systems built in real-time Linux [available at www.rtxi.org (Dorval et al., 2001; Raikov et al., 2004)] and LabVIEW (Kullmann et al., 2004) guarantee real-time performance. Systems built in Windows (Nowotny et al., 2006; Milescu et al., 2008) do not make this guarantee but can be shown to perform well for many applications (Milescu et al., 2008).

\section{Model error}

In most dynamic clamp experiments, the greatest source of inaccuracy stems from model error, which we define as a mistake in the form, parameters, or implementation of a modeled process. In virtual pharmacology experiments, an accurate knockin is predicated upon the assumption that the model truthfully captures the properties of the native channel. Aside from the inherent imperfections of voltage clamp measurements (Johnston and Brown, 1983; Williams and Mitchell, 2008; Sakmann and Neher, 2009), channel kinetics, densities, and voltage dependence have been shown to depend strongly on the neuromodulatory state of the neuron (Zhang and Harris-Warrick, 1995; Peck et al., 2006) as well as intracellular (Jensen et al., 2005) and extracellular (Zhang et al., 2001) concentrations of ions and other molecules. Model errors can even be introduced purposefully to formulate computationally efficient models of biophysical properties. For example, many channel models operate under the convenient but inaccurate assumption that activation and inactivation are independent (Hille, 2001). It is essentially certain that any voltage-gated conductance added via dynamic clamp will not perfectly mimic its naturally occurring counterpart, although the magnitude and importance of such model error is likely to vary considerably from case to case. A recent study aimed at minimizing this source of error uses online estimation to tune model parameters to account for cell-to-cell variation in channel properties (Milescu et al., 2008). This approach should prove valuable for optimizing channel models knocked in with virtual pharmacology.

Model error can appear in implementations of synaptic bombardment as well. A common method for simulating in vivolike background synaptic activity is to determine arrival times according to a random Poisson process. These processes are simple to implement computationally and incorporate the added benefit that the $\mathrm{SD}$ of interevent intervals is equal to the mean interval regardless of the rate at which synaptic inputs arrive. This is an important consideration for those attempting to separate the complementary effects of noise and increased membrane conductance introduced by in vivo-like activity on neuronal dynamics (Chance et al., 2002; Prescott and De Koninck, 2003; Tateno and Robinson, 2006). A particularly efficient approximation of the Poisson process is to determine the probability of an event occurring at each time step and then to apply a conductance transient via dynamic clamp according to that probability (Fernandez and White, 2008; Chance and Abbott, 2009). However, this approximation only holds if the rate of synaptic inputs is sufficiently small compared with the update rate of the dynamic clamp software. As a rule of thumb, the probability of an event occurring at each time step must be $<0.1$ to ensure that the ratio of the $\mathrm{SD}$ to the mean remains above 0.95 (M. N. Economo and J. A. White, unpublished observations). If this approximation does not hold, one must apply an appropriate correction (e.g., calculating the probability that two or more events occur in one interval) to avoid introducing unnecessary confounds into experimental results.

\section{Issues related to electrode-mediated conductance application}

Currents injected via dynamic clamp are typically introduced at the soma, as in conventional current clamp experiments. For this reason, virtual conductances mimicked by dynamic clamp are restricted to a single location in the membrane-the location of the recording pipette- unlike native conductances, which are distributed throughout the plasma membrane of the cell either uniformly or with some spatially varying density (Häusser et al., 2000; Stuart et al., 2007). Many of the effects of dendritic filtering can be inferred from somatic recordings of synaptic events, and modeling studies have indicated that the effects of somatic and dendritic synaptic barrages on the neuronal integrative properties are similar (Destexhe et al., 2003) (F. R. Fernandez and J. A. White, unpublished data). Nevertheless, it is clear that nonlinear interactions among synaptic inputs arriving at dendritic locations will not be captured using the traditional dynamic clamp approach. Additionally, changes in membrane conductance effected at the soma will not pervade the dendritic tree but are restricted to less than one space constant from the recording site (Williams, 2004).
One last notable aspect of conductances introduced with dynamic clamp is the necessary constraint that all current flowing into or out of the cell is mediated by the flux of a single charge carrier, typically the chloride ion. For this reason, chemical effects, such as the accumulation of calcium inside the cell following activation of calcium-permeable channels or dynamics associated with secondmessenger systems, cannot currently be captured via dynamic clamp, although such effects can be simulated (Feng and Jaeger, 2008).

\section{Conclusion}

We have reviewed several uses and some of the potential pitfalls of the dynamic clamp technique with the goal of helping readers understand and evaluate articles that use this emerging technique. The dynamic clamp technique allows electrophysiologists to test hypotheses in novel and powerful ways. In particular, dynamic clamp experiments are the only way to study the effects of mathematically precise manipulations of voltage- or ligand-gated channels on cellular integration in living neurons. For this reason, we expect use of the technique to grow.

With regard to the pitfalls of dynamic clamp, the one that prompts the most discussion is the difficulty in representing spatially extended conductances using a "point-source" electrode. This is a legitimate point that should always be kept in mind in interpreting dynamic clamp results, but this problem can be mitigated in many cases. For example, many studies (Chance et al., 2002; Netoff et al., 2005; Wolfart et al., 2005; Dorval and White, 2006; Prescott et al., 2008) focus on the effects of conductance-based synaptic input on neuronal integration at the soma and axon hillock. In most such studies, synapses are modeled using data from somatic recordings that include the effects of dendritic filtering. Thus, to the degree that the inputs sum linearly in the dendrites, the effects of the dendrite can be accounted for. Other techniques, including dendritic patching, real-time triggering of nerve shock, or neurotransmitter uncaging, can account for spatial locations as well. In evaluating this issue, the reader should keep in mind that we as a community generally accept results from somatic current-clamp experiments at face value, although this technique relies on a point source as well.

The problem of model error, which we define as systematic error in the details of simulated channels or input processes, is 
perhaps underappreciated by the neuroscience community at large. As in pure computational studies, it is essentially impossible to construct the perfect dynamic clamp representation of a set of channels, particularly voltage-gated channels. In both computational and dynamic clamp studies, one must demonstrate, or hope, that the effects of model error are minimal, which is often the case. Methods for automated tuning of model parameters to match control data (Milescu et al., 2008) may prove helpful in mitigating this problem.

We consider the future of the dynamic clamp technique bright. Nearly turnkey implementations are becoming more widespread. A number of courses in computational neuroscience (e.g., at the Marine Biological Laboratory, in Okinawa, Japan, and the Program of European Neuroscience Schools course in Europe) are increasing the level of awareness of the technique and pool of potential users at a dramatic pace. And the problems of spatial distributions of channels and model error can be mitigated. We hope that dynamic clamp becomes an integral part of every electrophysiologist's experimental toolbox.

\section{References}

Atallah BV, Scanziani M (2009) Instantaneous modulation of gamma oscillation frequency by balancing excitation with inhibition. Neuron 62:566-577.

Baranyi A, Szente MB, Woody CD (1993) Electrophysiological characterization of different types of neurons recorded in vivo in the motor cortex of the cat. II. Membrane parameters, action potentials, current-induced voltage responses and electrotonic structures. J Neurophysiol 69:1865-1879.

Bartos M, Manor Y, Nadim F, Marder E, Nusbaum MP (1999) Coordination of fast and slow rhythmic neuronal circuits. J Neurosci 19:6650-6660.

Berg RW, Alaburda A, Hounsgaard J (2007) Balanced inhibition and excitation drive spike activity in spinal half-centers. Science 315: 390-393.

Berg RW, Ditlevsen S, Hounsgaard J (2008) Intense synaptic activity enhances temporal resolution in spinal motoneurons. PLoS One 3:e3218.

Bettencourt JC, Lillis KP, Stupin LR, White JA (2008) Effects of imperfect dynamic clamp: computational and experimental results. J Neurosci Methods 169:282-289.

Borg-Graham LJ, Monier C, Frégnac Y (1998) Visual input evokes transient and strong shunting inhibition in visual cortical neurons. Nature 393:369-373.

Brette R, Piwkowska Z, Rudolph M, Bal T, Destexhe A (2007) A non-parametric electrode model for intracellular recording. Neurocomputing 70:1597-1601.

Brette R, Piwkowska Z, Monier C, Rudolph-Lilith
M, Fournier J, Levy M, Frégnac Y, Bal T, Destexhe A (2008) High-resolution intracellular recordings using a real-time computational model of the electrode. Neuron 59:379-391.

Butera RJ, McCarthy ML (2004) Analysis of real-time numerical integration methods applied to dynamic clamp experiments. J Neural Eng 1:187-194.

Chance FS, Abbott LF (2009) Simulating in vivo background activity in a slice with the dynamic clamp. In: Dynamic-clamp: from principles to applications (Destexhe A, Bal T eds), pp 73-87. New York: Springer.

Chance FS, Abbott LF, Reyes AD (2002) Gain modulation from background synaptic input. Neuron 35:773-782.

Destexhe A, Bal T (2009) Dynamic-clamp: from principles to applications, Ed 1. New York: Springer.

Destexhe A, Paré D (1999) Impact of network activity on the integrative properties of neocortical pyramidal neurons in vivo. J Neurophysiol 81:1531-1547.

Destexhe A, Rudolph M, Fellous JM, Sejnowski TJ (2001) Fluctuating synaptic conductances recreate in vivo-like activity in neocortical neurons. Neuroscience 107:13-24.

Destexhe A, Rudolph M, Paré D (2003) The high-conductance state of neocortical neurons in vivo. Nat Rev Neurosci 4:739-751.

Dorval AD Jr, White JA (2005) Channel noise is essential for perithreshold oscillations in entorhinal stellate neurons. J Neurosci 25:1002510028.

Dorval AD, White JA (2006) Synaptic input statistics tune the variability and reproducibility of neuronal responses. Chaos 16:026105.

Dorval AD, Christini DJ, White JA (2001) Realtime linux dynamic clamp: a fast and flexible way to construct virtual ion channels in living cells. Ann Biomed Eng 29:897-907.

Elson RC, Selverston AI, Abarbanel HD, Rabinovich MI (2002) Inhibitory synchronization of bursting in biological neurons: dependence on synaptic time constant. J Neurophysiol 88:1166-1176.

Fellous JM, Sejnowski TJ (2003) Regulation of persistent activity by background inhibition in an in vitro model of a cortical microcircuit. Cereb Cortex 13:1232-1241.

Feng SS, Jaeger D (2008) The role of SK calciumdependent potassium currents in regulating the activity of deep cerebellar nucleus neurons: a dynamic clamp study. Cerebellum 7:542-546.

Fernandez FR, White JA (2008) Artificial synaptic conductances reduce subthreshold oscillations and periodic firing in stellate cells of the entorhinal cortex. J Neurosci 28:3790-3803.

Goaillard J, Marder E (2006) Dynamic clamp analyses of cardiac, endocrine, and neural function. Physiology (Bethesda) 21:197-207.

Harris-Warrick RM, Coniglio LM, Levini RM, Gueron S, Guckenheimer J (1995) Dopamine modulation of two subthreshold currents produces phase shifts in activity of an identified motoneuron. J Neurophysiol 74: 1404-1420.

Häusser M, Spruston N, Stuart GJ (2000) Diversity and dynamics of dendritic signaling. Science 290:739-744.
Hille B (2001) Ion channels of excitable membranes, Ed 3. Sunderland, MA: Sinauer.

Hughes SW, Cope DW, Crunelli V (1998) Dynamic clamp study of Ih modulation of burst firing and delta oscillations in thalamocortical neurons in vitro. Neuroscience 87:541-550.

Idoux E, Serafin M, Fort P, Vidal PP, Beraneck M, Vibert N, Mühlethaler M, Moore LE (2006) Oscillatory and intrinsic membrane properties of guinea pig nucleus prepositus hypoglossi neurons in vitro. J Neurophysiol 96:175-196.

Jensen HS, Callø K, Jespersen T, Jensen BS, Olesen SP (2005) The KCNQ5 potassium channel from mouse: a broadly expressed M-current like potassium channel modulated by zinc, $\mathrm{pH}$, and volume changes. Brain Res Mol Brain Res 139:52-62.

Johnston D, Brown TH (1983) Interpretation of voltage-clamp measurements in hippocampal neurons. J Neurophysiol 50:464-486.

Jörntell H, Ekerot CF (2006) Properties of somatosensory synaptic integration in cerebellar granule cells in vivo. J Neurosci 26:1178611797.

Kullmann PH, Wheeler DW, Beacom J, Horn JP (2004) Implementation of a fast 16-Bit dynamic clamp using LabVIEW-RT. J Neurophysiol 91:542-554.

Matsumura M, Cope T, Fetz EE (1988) Sustained excitatory synaptic input to motor cortex neurons in awake animals revealed by intracellular recording of membrane potentials. Exp Brain Res 70:463-469.

Milescu LS, Yamanishi T, Ptak K, Mogri MZ, Smith JC (2008) Real-time kinetic modeling of voltage-gated ion channels using dynamic clamp. Biophys J 95:66-87.

NetoffTI, Banks MI, Dorval AD, Acker CD, Haas JS, Kopell N, White JA (2005) Synchronization in hybrid neuronal networks of the hippocampal formation. J Neurophysiol 93:1197-1208.

Nowotny T, Szucs A, Pinto RD, Selverston AI (2006) StdpC: a modern dynamic clamp. J Neurosci Methods 158:287-299.

Paré D, Shink E, Gaudreau H, Destexhe A, Lang EJ (1998) Impact of spontaneous synaptic activity on the resting properties of cat neocortical pyramidal neurons In vivo. J Neurophysiol 79:1450-1460.

Peck JH, Gaier E, Stevens E, Repicky S, HarrisWarrick RM (2006) Amine modulation of Ih in a small neural network. J Neurophysiol 96:2931-2940.

Prescott SA, De Koninck Y (2003) Gain control of firing rate by shunting inhibition: roles of synaptic noise and dendritic saturation. Proc Natl Acad Sci U S A 100:2076-2081.

Prescott SA, Ratté S, De Koninck Y, Sejnowski TJ (2006) Nonlinear interaction between shunting and adaptation controls a switch between integration and coincidence detection in pyramidal neurons. J Neurosci 26:9084-9097.

Prescott SA, Ratté S, De Koninck Y, Sejnowski TJ (2008) Pyramidal neurons switch from integrators in vitro to resonators under in vivo-like conditions. J Neurophysiol 100:3030-3042.

Preyer AJ, Butera RJ (2009) Causes of transient instabilities in the dynamic clamp. IEEE Trans Neural Syst Rehabil Eng 17:190-198.

Prinz AA, Thirumalai V, Marder E (2003) The 
functional consequences of changes in the strength and duration of synaptic inputs to oscillatory neurons. J Neurosci 23:943-954.

Raikov I, Preyer A, Butera RJ (2004) MRCI: a flexible real-time dynamic clamp system for electrophysiology experiments. J Neurosci Methods 132:109-123.

Sakmann B, Neher E (2009) Single-channel recording. New York: Springer.

Sharp AA, O’Neil MB, Abbott LF, Marder E (1993) The dynamic clamp: artificial conductances in biological neurons. Trends Neurosci 16:389-394.

Sharp AA, Skinner FK, Marder E (1996) Mechanisms of oscillation in dynamic clamp constructed two-cell half-center circuits. J Neurophysiol 76:867-883.

Sohal VS, Zhang F, Yizhar O, Deisseroth K (2009) Parvalbumin neurons and gamma rhythms enhance cortical circuit performance. Nature 459:698-702.

Stuart G, Spruston N, Häusser M (2007) Dendrites, Ed 2. Oxford: Oxford UP.
Tateno T, Robinson HP (2006) Rate coding and spike-time variability in cortical neurons with two types of threshold dynamics. J Neurophysiol 95:2650-2663.

Thirumalai V, Prinz AA, Johnson CD, Marder E (2006) Red pigment concentrating hormone strongly enhances the strength of the feedback to the pyloric rhythm oscillator but has little effect on pyloric rhythm period. J Neurophysiol 95:1762-1770.

Traub RD, Whittington MA, Colling SB, Buzsáki G, Jefferys JG (1996) Analysis of gamma rhythms in the rat hippocampus in vitro and in vivo. J Physiol 493:471-484.

Vervaeke K, Hu H, Graham LJ, Storm JF (2006) Contrasting effects of the persistent $\mathrm{Na}+$ current on neuronal excitability and spike timing. Neuron 49:257-270.

Whittington MA, Traub RD, Kopell N, Ermentrout B, Buhl EH (2000) Inhibition-based rhythms: experimental and mathematical observations on network dynamics. Int J Psychophysiol 38:315-336
Wilders R (2006) Dynamic clamp: a powerful tool in cardiac electrophysiology. J Physiol 576:349-359.

Williams SR (2004) Spatial compartmentalization and functional impact of conductance in pyramidal neurons. Nat Neurosci 7:961-967.

Williams SR, Mitchell SJ (2008) Direct measurement of somatic voltage clamp errors in central neurons. Nat Neurosci 11:790-798.

Wolfart J, Debay D, Le Masson G, Destexhe A, Bal T (2005) Synaptic background activity controls spike transfer from thalamus to cortex. Nat Neurosci 8:1760-1767.

Zhang B, Harris-Warrick RM (1995) Calciumdependent plateau potentials in a crab stomatogastric ganglion motor neuron. I. Calcium current and its modulation by serotonin. J Neurophysiol 74:1929-1937.

Zhang S, Kehl SJ, Fedida D (2001) Modulation of Kv1.5 potassium channel gating by extracellular zinc. Biophys J 81:125-136. 